\title{
AKR1C1-3, notably AKR1C3, are distinct biomarkers for liver cancer diagnosis and prognosis: Database mining in malignancies
}

\author{
SHOU-FENG ZHAO ${ }^{1}$, SHU-GUO WANG ${ }^{2}$, ZI-YUN ZHAO ${ }^{3}$ and WEN-LI LI ${ }^{4}$ \\ ${ }^{1}$ Central Laboratories; ${ }^{2}$ Department of Clinical Laboratory, Qingdao Municipal Hospital, Qingdao, \\ Shandong 266000; ${ }^{3}$ Department of Laboratory Medicine, Qingdao Central Hospital, Qingdao, Shandong 266044; \\ ${ }^{4}$ Department of Gastroenterology, Qingdao Municipal Hospital, Qingdao, Shandong 266000, P.R. China
}

Received June 20, 2018; Accepted July 12, 2019

DOI: $10.3892 / 01.2019 .10802$

\begin{abstract}
Aldo-keto reductases, known as AKR1C1-AKR1C4 enzymes, are pivotal to NADPH-dependent reduction, and their expression is highly associated with the progression of malignant cancers. However, the expression and distinct prognostic value of the AKR1C family members in liver cancer are not well established. In the current study, the expression of AKR1C isoforms was studied using the Oncomine online databases. In addition, their expression profiles were analyzed in cancer cell lines using data from the Cancer Cell lines Encyclopedia (CCLE) database. Furthermore, the mRNA expression levels of AKR1C family members between liver cancer and normal liver samples were assessed by the Gene Expression Profiling Interactive Analysis (GEPIA) database. The AKR1C1-3 prognostic value was further investigated by the Kaplan-Meier plotter database in liver cancer patients. It was found that the expression levels of AKR1C3 were elevated significantly in liver cancer tissues and cells as demonstrated by the Oncomine, CCLE and GEPIA databases. The expression levels of AKR1C1 and AKR1C2 in liver cancer tissues did not increase significantly in the Oncomine database while expression was significantly high in CCLE and GEPIA databases. However, the expression levels of the AKR1C4 gene as determined by the CCLE, GEPIA and Oncomine databases were not consistent. Therefore, the Kaplan-Meier survival curves of liver cancer patients with the expression of AKR1C1-3 genes were next analyzed. The data indicated that high expression levels of AKR1C1-3 were correlated with lower overall survival in liver cancer patients. Using the co-expression and PPI network, AKRIC1-3 genes were identified that were involved in the same pathway displaying 44 total unique interactors. These results suggested that the increased
\end{abstract}

Correspondence to: $\mathrm{Dr}$ Wen-Li Li, Department of Gastroenterology, Qingdao Municipal Hospital, 1 Jiaozhou Road, Qingdao, Shandong 266000, P.R. China

E-mail: 1w1201@163.com

Key words: liver cancer, AKR1C family members, biomarker, prognostic factor, database mining
AKR1C1-3, notably AKR1C3 expression levels served as possible diagnostic biomarkers and essential prognostic factors for liver cancer patients. The roles of AKR1C4 in liver cancer require further examination.

\section{Introduction}

According to the cancer statistics database from the American Cancer Society (ACS) liver cancer is one of the six most frequent malignancies and ranks the fifth leading cause of cancer-associated mortalities in the US (1). Globally, liver cancer is the 3rd leading cause of cancer-related death with $\sim 700,000$ deaths occurring annually (2). Despite the combined use of chemotherapy and surgery, the prognosis and survival rates of liver cancer patients remain poor (3).

The early stage diagnosis of liver cancer and the prediction of liver cancer patient prognosis are considered imperative for the prevention of this disease (4). Certain tumor markers including $\alpha$-fetoprotein, carcinoembryonic antigen, transforming growth factor- $\beta$ and microRNA-500/29/112 have been previously used for early diagnosis and prognosis of liver cancer $(5,6)$. However, the use of these tumor markers remains limited. Therefore, several research groups have focused on the development of novel biomarkers for the early detection and prognosis of liver cancer.

$\operatorname{NAD}(\mathrm{P})(\mathrm{H})$-dependent oxidoreductase genes of the aldo-keto reductase type $1 \mathrm{C}(A K R 1 C)$ family, including $A K R 1 C 1, A K R I C 2, A K R I C 3$ and AKRIC4 are located on chromosome 10p15-p14 (7). Normally, AKR1C1, AKR1C2, AKR1C3 and AKR1C4 enzymes are implicated in steroid metabolism and their expression levels are localized in the normal tissues of the lung, liver, prostate, testis and mammary glands. The upregulation or downregulation of AKR1C1, AKR1C2, AKR1C3 and AKR1C4 are associated with several human tumors including ovarian, breast, colon, prostate, gastric and leukemia (8-12). However, the exact functions of the different AKR1C1, AKR1C2, AKR1C3 and AKR1C4 members in combination with tumorigenesis and liver cancer prognosis have not been fully identified.

In the present study, the expression levels of the AKRIC family isoforms were examined in liver cancer, in order to determine the expression pattern of distinct AKR1C family members in liver cancer tissues compared with that noted in 
normal tissues. Moreover, the corresponding prognostic value of AKR1C1-3 was examined in liver cancer. The present analysis can improve the early diagnosis of liver cancer and support the individual care for liver cancer treatment.

\section{Materials and methods}

Validation AKRIC1, AKRIC2, AKRIC3 and AKRIC4 genes expression by Oncomine analysis. Oncomine gene expression array datasets (https://www.oncomine.org/) is an online cancer microarray database (13) that contains 19 cancer types, 715 datasets and 86,733 samples corresponding to $\sim 48$ million gene expression measurements. Differential expression analyses comparing most cancer tissue types with the corresponding normal tissues were available for exploration. The data could be queried and visualized for a selected gene across all analyses (13). Regarding the differential analysis of AKRIC1, AKRIC2, AKRIC3 and AKRIC4 between liver cancer tissues and normal liver samples, the thresholds were set as follows: Analysis type: Cancer vs. normal; cancer type: Liver cancer; sample type: Clinical specimen; data type: mRNA. The individual mRNA levels of AKRICl, AKRIC2, $A K R I C 3$ and AKRIC4 in different types of cancers were determined from the Oncomine database in comparison with the levels of the corresponding normal tissues. In the present study, the top $10 \%$ gene rank with a $\mathrm{P}<0.001$ and fold-change $>2$ were used as thresholds.

Evaluation of AKR1C1, AKRIC2, AKRIC3 and AKRIC4 $m R N A$ levels by Cancer Cell lines Encyclopedia (CCLE) analysis. The CCLE project is a collaboration between the Broad Institute, the Novartis Institutes for Biomedical Research and the Genomics Novartis Foundation that aims to conduct a detailed genetic and pharmacological characterization of a large panel of human cancer models in order to develop integrated computational analyses that link distinct pharmacological vulnerabilities to genomic patterns (14). Furthermore, the CCLE project aims to translate cell line integrative genomics into cancer patient stratification and consists of a compilation of gene expression, chromosomal copy number and parallel sequencing data (14). All raw and processed data are available at an integrated portal on www. broadinstitute.org/ccle. The CCLE provides public access to genomic data, analysis and visualization for 1,062 cell lines representing 37 distinct cancer types. The investigation of the mRNA levels of AKRIC1, AKRIC2, AKRIC3 and AKRIC4 in a series of cancer cell lines was conducted by CCLE analysis. The gene expression data were derived from human cancer cell lines, in order to identify whether and/or to what extent the AKRIC1, AKRIC2, AKRIC3 and AKRIC4 genes were expressed in liver cancer cell lines compared with their expression in other types of cancer cell lines.

Comparison of the AKRIC family gene expression levels in liver cancer and paired normal tissues by the Gene Expression Profiling Interactive Analysis (GEPIA) database. GEPIA is a newly developed interactive web database including 9,736 tumor and 8,587 normal samples from the TCGA and GTEx projects, which can be used for RNA sequencing and RNA expression analyses. GEPIA provides customizable functions, such as tumor and/or normal differential expression analysis, profiling according to cancer types or pathological stages, patient survival analysis, detection of gene expression similarities, correlation analysis and dimensionality reduction analysis. GEPIA fills in the gap between cancer genomics big data and the delivery of integrated information to end users, thereby facilitating the prognostic value of the current data resources. GEPIA is available at http://gepia.cancer-pku.cn/. In the present study, this online tool was used to analyze the levels of the AKRIC family members between liver cancer and normal control specimens. Statistical analysis was performed using the Student's t-test to and the cut-off $\mathrm{P}<0.01$.

Prognostic value assessment of AKRIC1-3 using the Kaplan-Meier plotter survival analysis. The prognostic significance of the mRNA expression levels of the AKRIC1-3 in several cancer types was evaluated using the Kaplan-Meier plotter (http://www.kmplot.com), an online database that can be used to assess the effect of genes in cancer prognosis. The Kaplan-Meier plotter can assess the effects of 54,675 genes on survival using 10,461 cancer samples. The analysis was applied to evaluate the prognostic value of the gene expression levels of the AKRIC1-3 in liver cancer. In this database, the data were extracted regarding lung (15), ovarian (16), liver (17), gastric (18) and breast cancers (19). The patient samples were divided into two cohorts according to the median expression of each gene (high vs. low expression). The overall survival (OS) of liver cancer patients was analyzed using a Kaplan-Meier survival plot. Briefly, the three genes (AKRICl, AKRIC2 and $A K R I C 3)$ were uploaded into the database to obtain the Kaplan-Meier survival plots, in which the number-at-risk was shown below the main plot. Subsequently, the plot data was exported as text and the Kaplan-Meier survival curves for the expression of each gene of interest.

Co-expression and protein-protein interaction (PPI) network construction. The top 100 co-expression genes were extracted of AKRIC3 across all tumor samples from the GEPIA database (http://gepia.cancerpku.cn/index.html) and then the Search Tool for the Retrieval of Interacting Genes (STRING) database (http://string-db.org/) was designed in order to analyze the PPI information (20). To evaluate the potential PPI relationship, the previously identified DEGs were mapped to the STRING database. The PPI pairs were extracted with a combined score of 0.4. Subsequently, the PPI network was visualized by the Cytoscape software (version 3.6.1; www.cytoscape.org/). The nodes with a higher degree of connectivity appeared more essential in maintaining the stability of the entire network.

Statistical analysis. AKRICl-4 genes were queried in Oncomine database and the results were filtered by selecting 'liver cancer', 'mRNA and cancer vs. normal analysis', '2-fold change', ' $\mathrm{P}<0.001$ ' and 'top $10 \%$ gene rank'. In CCLE database, the line within a box indicatesthe mean. The following settings for GEPIA were used as: 'Expression on Box Plots', 'Gene=FNDC1', ' $\log 2 \mathrm{FCl}$ Cutoff=1', 'P-value Cutoff $=0.01$ ', 'Datasets $=$ STAD', 'Log Scale $=\log _{2}(\mathrm{TPM}+1)$, ' Jitter Size $=0.4$ ' and 'Match TCGA normal and GTEx data'. The prognostic value of the AKRIC1-3 genes in liver cancer was analyzed using the Kaplan-Meier Plotter. The settings were used for the 
analysis: 'Overall survival', 'auto select best cutoff', 'censored at threshold', (patients surviving over the selected threshold are censored instead of excluded), 'tumor stage all', 'tumor stage Tall', 'tumor stage $\mathrm{N}$ all', 'tumor stage $\mathrm{M}$ all', 'Lauren classification all' and 'differentiation all'. The $A K R 1 C$ genes probe set was 48-315996, 17-101636, 226-101485 and 226-101485 respectively. Patients were split according to median expression or expression at best cutoff for the probe. The results of the Kaplan-Meier analysis are presented with hazard ratio (HR) and log-rank P-values from a log-rank test. $\mathrm{P}<0.05$ was considered to indicate a statistically significant difference. The data were extracted from the Oncomine database, CCLE, GEPIA website and Kaplan-Meier Plotter between December 2017 and March 2019.

\section{Results}

The diverse expression patterns of AKR1C1, AKR1C2, $A K R I C 3$ and AKRIC4 genes as determined by analysis of the Oncomine database. Using Oncomine analysis, the mRNA levels of the AKRIC family of genes in various human cancer types including hematogenous malignancies and solid tumors were investigated. The number in each cell represents the number of analyses that meet the threshold within those analysis and cancer types. There were 3 and 2 analyses that meet the thresholds for AKR1C3 and AKR1C4 respectively (Fig. 1). No analysis met the thresholds for AKR1C1 and AKR1C2.

To further examine the expression status of $A K R 1 C 1$, $A K R 1 C 2, A K R 1 C 3$ and AKRIC4 genes in liver cancer, the data corresponding to the four genes with regard to the liver cancer tissue number, normal tissue number, fold-change, t-test T, P-value and rank were summarized (Table I). Among the $A K R I C$ family of genes, $A K R I C 3$ was overexpressed at the highest levels in liver cancer $(n=386)$ compared with those noted in normal tissues $(\mathrm{n}=327)$ in the studies of Chen et al (21), Wurmbach et al (22) and Roessler et al (23) with fold-changes between 1.774 and 3.438. The differential expression analysis of $A K R 1 C 1$ and $A K R 1 C 2$ exhibited fold-changes of 1.435 and 1.543 for liver cancer $(n=35)$ and normal tissues $(n=10)$, respectively, as determined in the Wurmbach et al (22) study. However, AKRIC4 was downregulated in liver cancer $(\mathrm{n}=225)$ compared with its corresponding expression in normal tissues $(n=220)$ with a fold change of -2.594 as shown in the Roessler et al (23) study.

The expression levels of $A K R 1 C 3$ were differentially overexpressed between liver cancer and normal tissues, whereas the expression levels of $A K R 1 C 4$ were downregulated in liver cancer tissues. The expression levels of $A K R I C 1$ and $A K R I C 2$ in liver cancer tissues were not increased significantly.

The expression levels of AKR1C1, AKRIC2, AKR1C3 and AKRIC4 are upregulated in liver cancer cell lines as determined by CCLE analysis. The expression levels of AKRIC1, $A K R 1 C 2, A K R 1 C 3$ and $A K R 1 C 4$ in liver cancer cell lines were ranked as the top 3 out of 37 distinct cancer types in the CCLE databases (Fig. 2). The total of their expression levels was higher than that of the other cancer types (Fig. 2). These results were consistent with those for AKRICl-3 indicating that these genes were upregulated in liver cancer as determined by the Oncomine database. In contrast to $A K R 1 C 1-3$, the expression
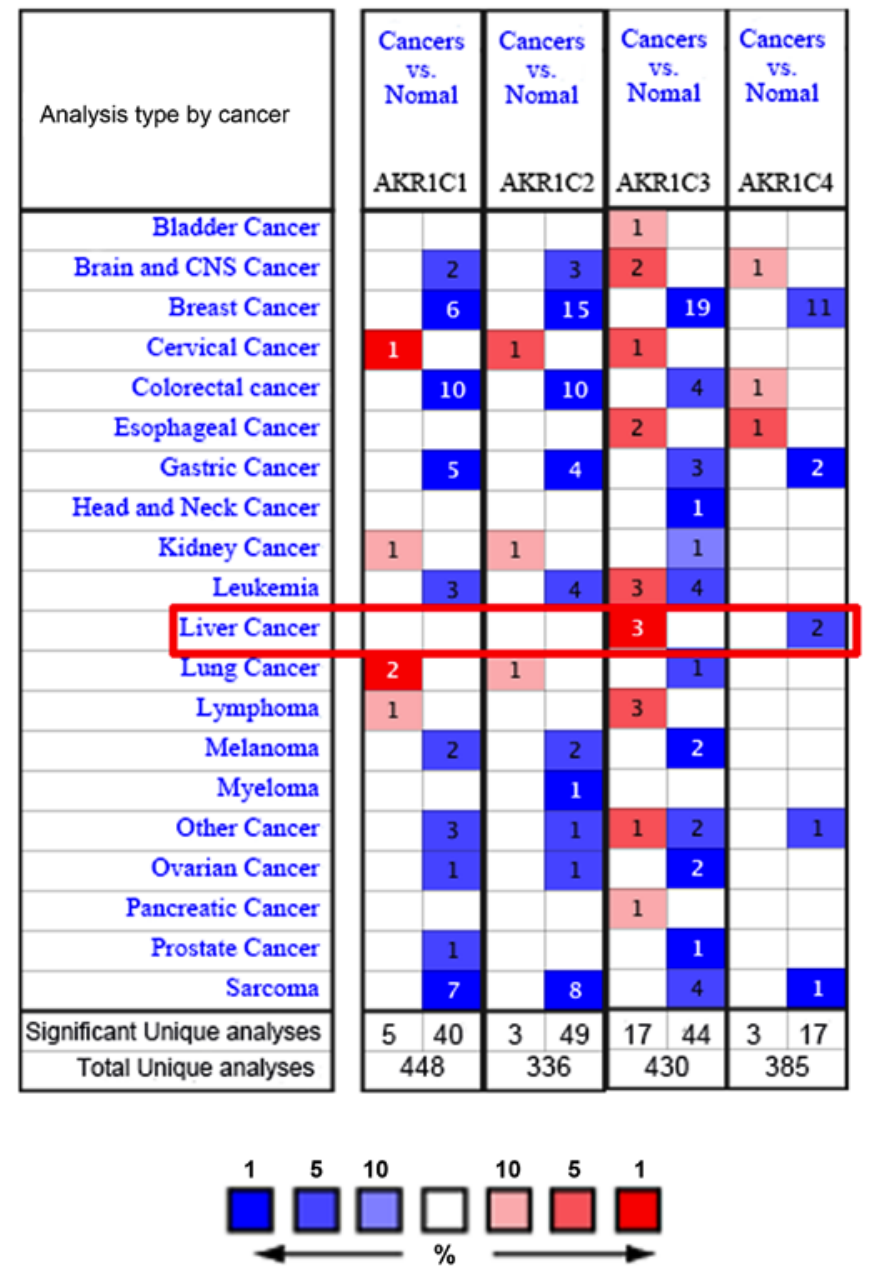

Figure 1. mRNA expression levels of $A K R 1 C 1, A K R 1 C 2, A K R 1 C 3$ and $A K R 1 C 4$ in cancers. The graphic demonstrates the numbers of datasets with statistically significantly upregulated (red) or downregulated (blue) expression of the target gene mRNA. The cell color is determined by the best gene rank percentile for the analyses within the cell. The number in each cell represents the number of analyses that met the criteria 'Gene: $A K R 1 C 1$ or AKR1C2 or AKR1C3 or AKR1C4; Analysis Type: Cancer vs. Normal Analysis; Cancer Type: Liver Cancer; Data Type: mRNA'. The expression levels of the four genes in liver cancer are shown in the red frame. The significant mRNA of the AKR1C3 and AKR1C4 genes were upregulated (red cell, 3 analyses) and downregulated (blue cell, 2 analysis), respectively, whereas the expression levels of $A K R 1 C 1$ and $A K R 1 C 2$ did not significantly change (white cell, no analysis). The gene rank was analyzed by the percentile of target gene in the top of all genes measured in each research. Data observed using Oncomine database (https://www.oncomine.org/). AKR1C, aldo-keto reductase type $1 \mathrm{C}$.

levels of $A K R 1 C 4$ were significantly downregulated in liver cancer samples derived from the Oncomine database but were highly expressed in the CCLE database (Table I; Fig. 1). It was implied that $A K R I C 1-3$, especially $A K R I C 3$ may play roles in the development of liver cancer. The role of $A K R 1 C 4$ in liver cancer should be further examined.

AKR1C1, AKR1C2, AKRIC3 and AKR1C4 are differentially expressed in liver cancer tissues as determined by GEPIA analysis. To examine the expression levels of $A K R I C$ members in liver cancer, their mRNA levels were compared between liver cancer and normal liver samples using GEPIA. The GEPIA database included 369 liver cancer and 160 normal liver tissues, which were used for the expression analysis (24). 
Table I. The mRNA expression of $A K R I C$ family genes in normal and liver cancer tissue.

\begin{tabular}{lcccccrc}
\hline Gene & Liver cancer, $\mathrm{n}$ & Normal, $\mathrm{n}$ & Fold-change & t-test, $\mathrm{T}$ & P-value & Gene rank $^{\mathrm{a}}, \%$ & (Refs.) \\
\hline AKR1C1 & 35 & 10 & 1.435 & 2.533 & $8.00 \times 10^{-3}$ & 21 & $(21)$ \\
AKR1C2 & 35 & 10 & 1.543 & 3.626 & $4.53 \times 10^{-4}$ & 11 & $(21)$ \\
AKR1C3 & 225 & 220 & 3.438 & 22.18 & $3.86 \times 10^{-71}$ & 1 & $(22)$ \\
& 22 & 21 & 2.965 & 6.185 & $1.44 \times 10^{-7}$ & 4 & $(22)$ \\
& 35 & 10 & 2.470 & 6.309 & $1.62 \times 10^{-6}$ & 3 & $(21)$ \\
AKR1C4 & 104 & 76 & 1.774 & 6.140 & $2.81 \times 10^{-9}$ & 7 & $(22)$ \\
\hline
\end{tabular}

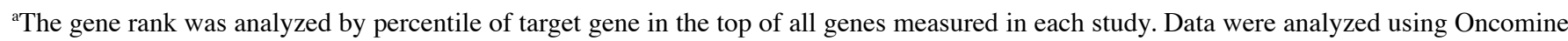
database (https://www.oncomine.org/). AKR1C, aldo-keto reductase type 1C.
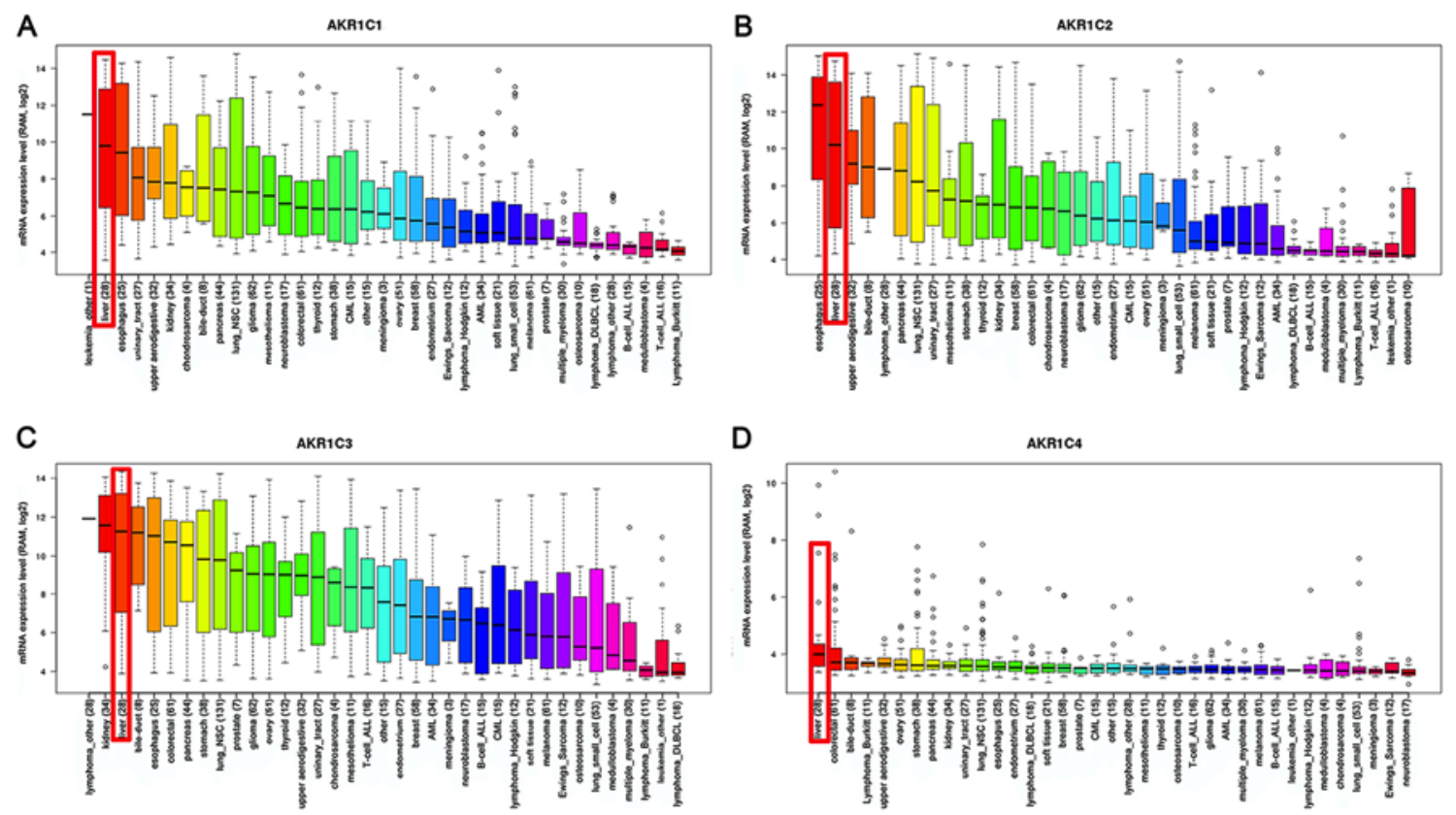

Figure 2. The expression levels of $A K R 1 C 1, A K R 1 C 2, A K R 1 C 3$ and $A K R 1 C 4$ were upregulated in liver cancer cell lines. (A-D) The mRNA expression levels of (A) $A K R 1 C 1$, (B) $A K R 1 C 2$, (C) $A K R 1 C 3$ and (D) AKRIC4 in liver cancer cell lines were ranked second, second, third and the first among a variety of cancer cell lines, respectively (shown in red frame). Data were analyzed using the Cancer Cell Line Encyclopedia database (www.broadinstitute.org/ccle). RMA, Robust Multi-array Average; AKRIC, aldo-keto reductase type 1C.

The data indicated that the $A K R I C 1-4$ gene members were overexpressed in liver cancer samples compared with normal liver samples. The boxplot $(\mathrm{P}<0.05$; Fig. 3$)$ revealed consistent data with those obtained by the CCLE database analysis.

Association of AKR1C1, AKR1C2 and AKR1C3 with survival time in liver cancer patients. It was found that the expression levels of AKR1C1-3 were elevated in liver cancer tissues and cells as demonstrated by the Oncomine, CCLE and GEPIA databases. However, the expression levels of the AKR1C4 gene as determined by the Oncomine, CCLE and GEPIA databases were not consistent. Therefore, the association between the liver cancer patient survival time and the mRNA expression levels of the AKRIC1-3 genes was identified using a Kaplan-Meier plotter. The survival curves were plotted for all liver cancer patients (Fig. 4). Liver cancer patients with high expression of AKR1C1 $(\mathrm{HR}=1.78 ; \mathrm{P}=0.0012), \mathrm{AKR} 1 \mathrm{C} 2(\mathrm{HR}=1.51 ; \mathrm{P}=0.028)$ and AKR1C3 $(\mathrm{HR}=1.69 ; \mathrm{P}=0.027)$ exhibited a significant association with lower OS. High mRNA expression levels of AKR1C1, AKR1C2 and AKR1C3 were significantly associated with low OS in all liver cancer patients.

The co-expression and PPI network construction. Co-expression gene analysis was conducted by the PPI networks of AKR1C3, which were differentially expressed in liver cancer (Fig. 5). It was shown that $A K R I C 1-3$ was involved in the same pathway displaying 44 total unique interactors.

\section{Discussion}

The aldo-keto reductase type $1 \mathrm{C}$ comprises the isoforms AKR1C1-AKR1C4 that serve important roles in the metabolism of steroid hormones, conjugated steroids, neurosteroids and bile acids (10,12,25-27). These enzymes are also involved 


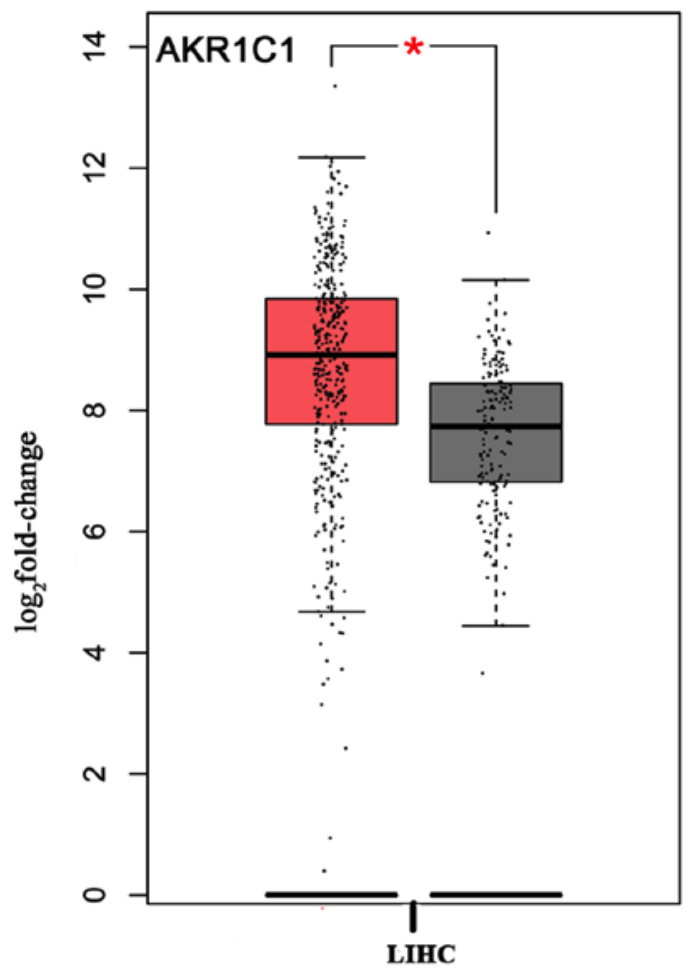

$(\operatorname{num}(T)=369 ; \operatorname{num}(\mathrm{N})=160$

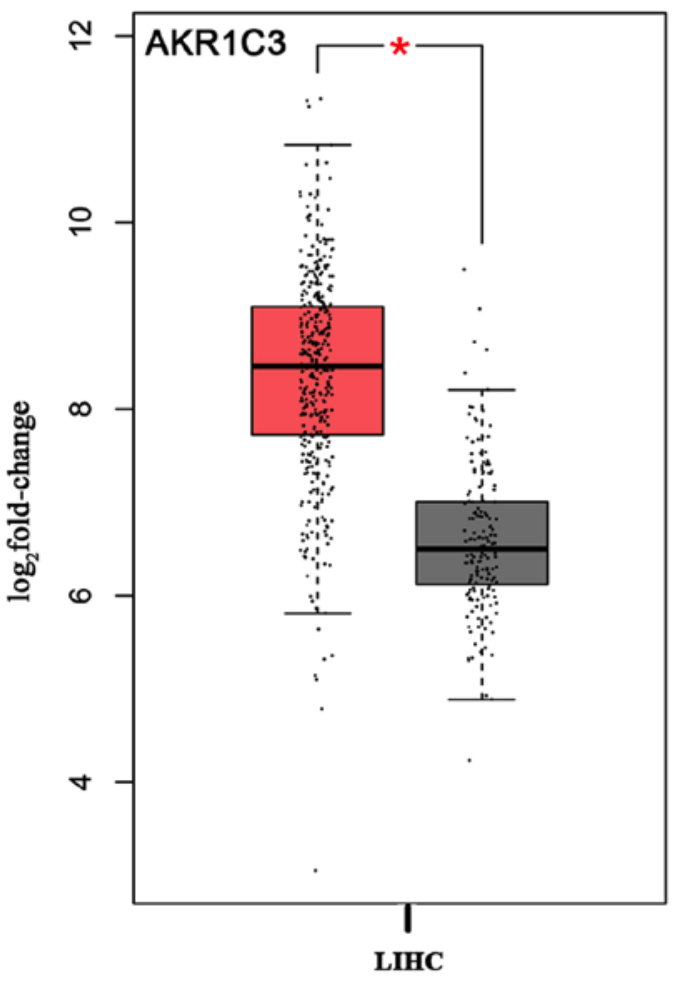

$(\operatorname{num}(\mathrm{T})=369 ; \operatorname{num}(\mathrm{N})=160$

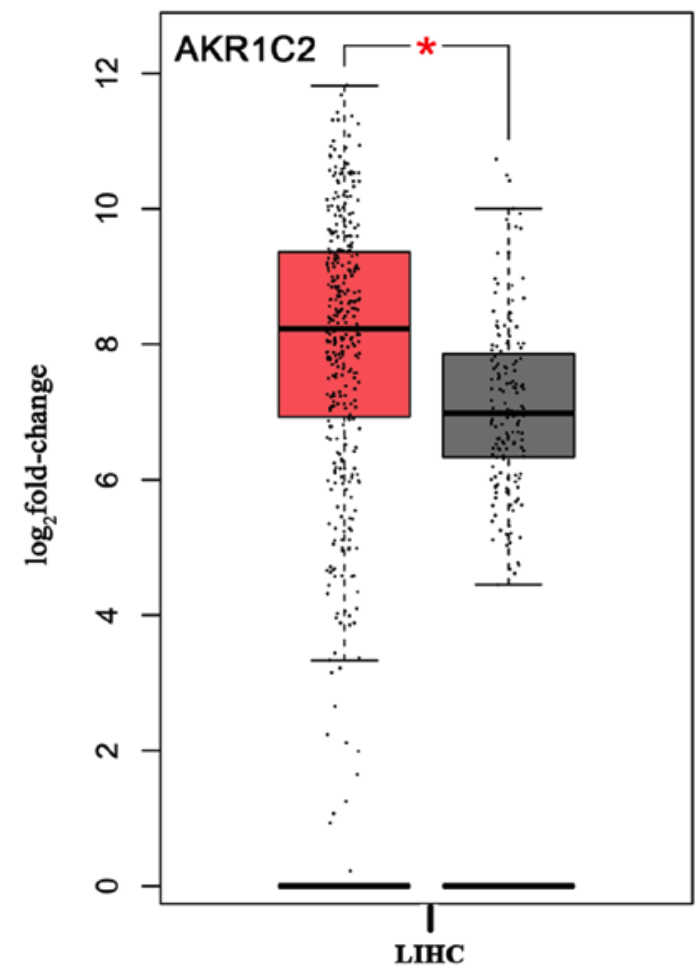

$(\operatorname{num}(T)=369 ; \operatorname{num}(N)=160$

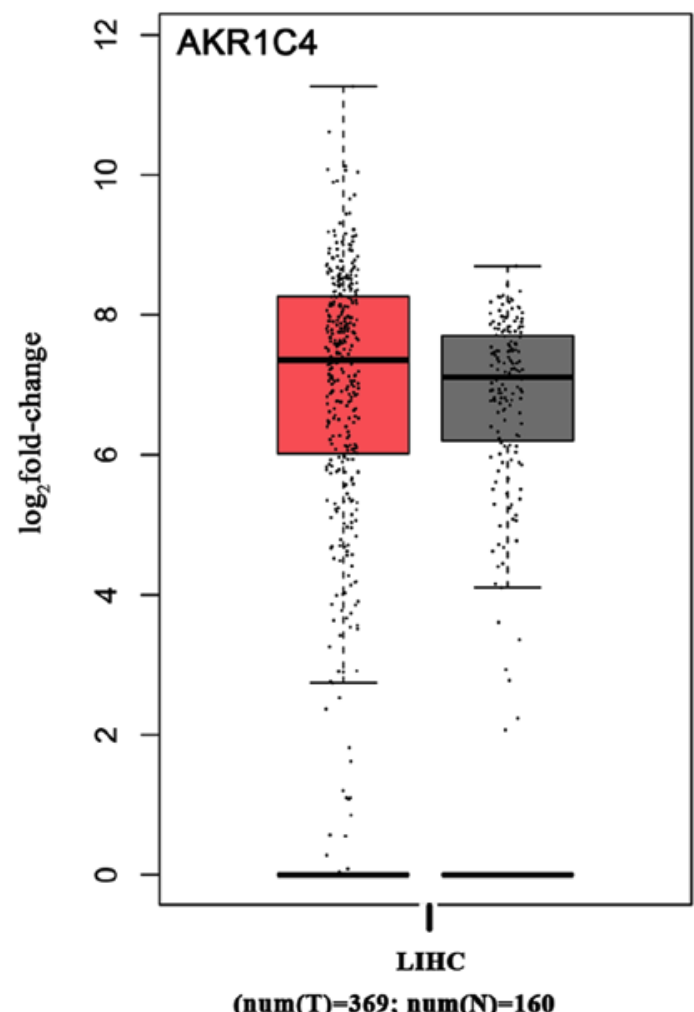

Figure 3. AKRIC1-4 are upregulated in liver cancer samples compared with normal liver samples. The box plots of 369 liver cancer samples (red) and 160 normal samples (gray) revealed upregulation of $A K R I C$ family members in liver cancer compared with normal tissue. The height of each bar represents the median expression of certain tumor or normal tissues. The whiskers represent the minimum and maximum values within each sample set. Data were analyzed using GEPIA database (http://gepia.cancer-pku.cn/). "P<0.05. LIHC, liver hepatocellular carcinoma. AKRIC, aldo-keto reductase type 1C.

in the modulation of carcinogen metabolism (10,12,25-27). AKR1C1 is an important contributor in the proliferation and migration of tumor cells including small-cell lung cancer and breast cancer $(12,26)$. The AKRIC2 gene may contribute to the incidence, progression and invasion of breast cancer (12) and hepatocellular carcinoma (28). AKRIC3 overexpression is associated with the progression and aggressiveness of non-small-cell lung cancer, esophageal cancer and prostate 

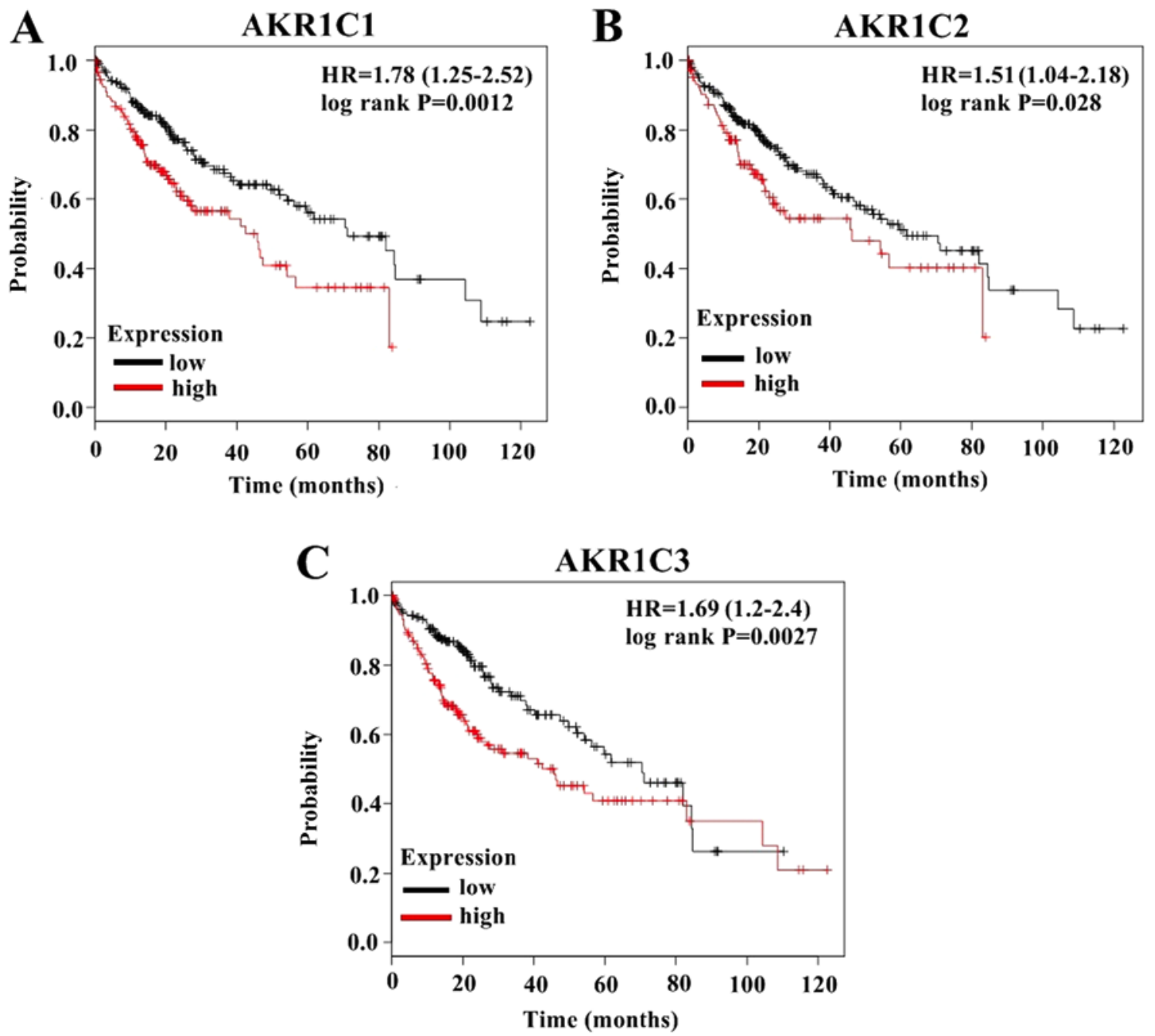

Figure 4. Patients with liver cancer with high expression of $A K R 1 C 1-3$ exhibit poor overall survival. (A-C) The survival curves of the (A) $A K R 1 C 1$, (B) $A K R 1 C 2$ and (C) $A K R 1 C 3$ expression analysis were plotted using Kaplan-Meier Plotter for patients with liver cancer. The red line indicates patients with AKRIC expression above the median, and the black line indicates patients with AKR1C factor expression below the median. Data were obtained from the Kaplan-Meier database (http://www.kmplot.com). AKRlC, aldo-keto reductase type 1C; HR, hazard ratio.

cancer $(10,29,30)$. The catalytic-dependent and-independent function of the AKR1C isoforms demonstrated critical roles in the proliferation and migration of cells and in tumor drug resistance (31). To the best of our knowledge, the association between the expression levels of the AKR1C isoforms and the incidence of liver cancer has not been examined to date.

Liver cancer is one of the leading causes of cancer-related deaths worldwide. The 5-year OS rate of liver cancer patients is $<5 \%$ (32). Early diagnosis provides the only cure for these patients. In addition to surgical resection, ablation and liver transplantation are used frequently as therapeutic modalities for liver cancer patients. Molecular-targeted therapies are considered potential emerging treatment of advanced liver cancer.

The present study initially demonstrated that high expression levels of $A K R 1 C 1-3$, notably $A K R 1 C 3$, predicted low survival. Using the Oncomine, CCLE and GEPIA databases, the expression levels of the $A K R I C$ family members were analyzed in liver cancer samples and compared with the corresponding expression levels of the normal samples. The results demonstrated that the expression levels of $A K R 1 C 3$ were elevated in liver cancer tissues compared with those of the normal tissues. The expression levels of AKR1C1 and AKR1C2 in liver cancer tissues were not increased significantly in the Oncomine database while expression was high in CCLE and GEPIA databases. However, the expression levels of the AKR1C4 gene that were noted in the Oncomine, CCLE and GEPIA databases were not consistent. The role of $A K R 1 C 4$ in liver cancer requires further examination. The elevated expression of $A K R I C 3$ in the Oncomine, CCLE and GEPIA databases suggested that $A K R 1 C 3$ may serve as a potential diagnostic, therapeutic biomarker for liver cancer patients.

Survival analysis of the levels of $A K R 1 C 1-3$ demonstrated similar results indicating that overexpression of $A K R 1 C 1-3$ was associated with lower survival in patients with liver cancer. These results suggested the critical role of AKR1C1-3 regarding their contribution in liver cancer initiation and/or progression.

To the best of our knowledge, the regulation of the expression levels of $A K R I C$ in liver cancer has not been explored. A limited number of reports have shown that the overexpression 


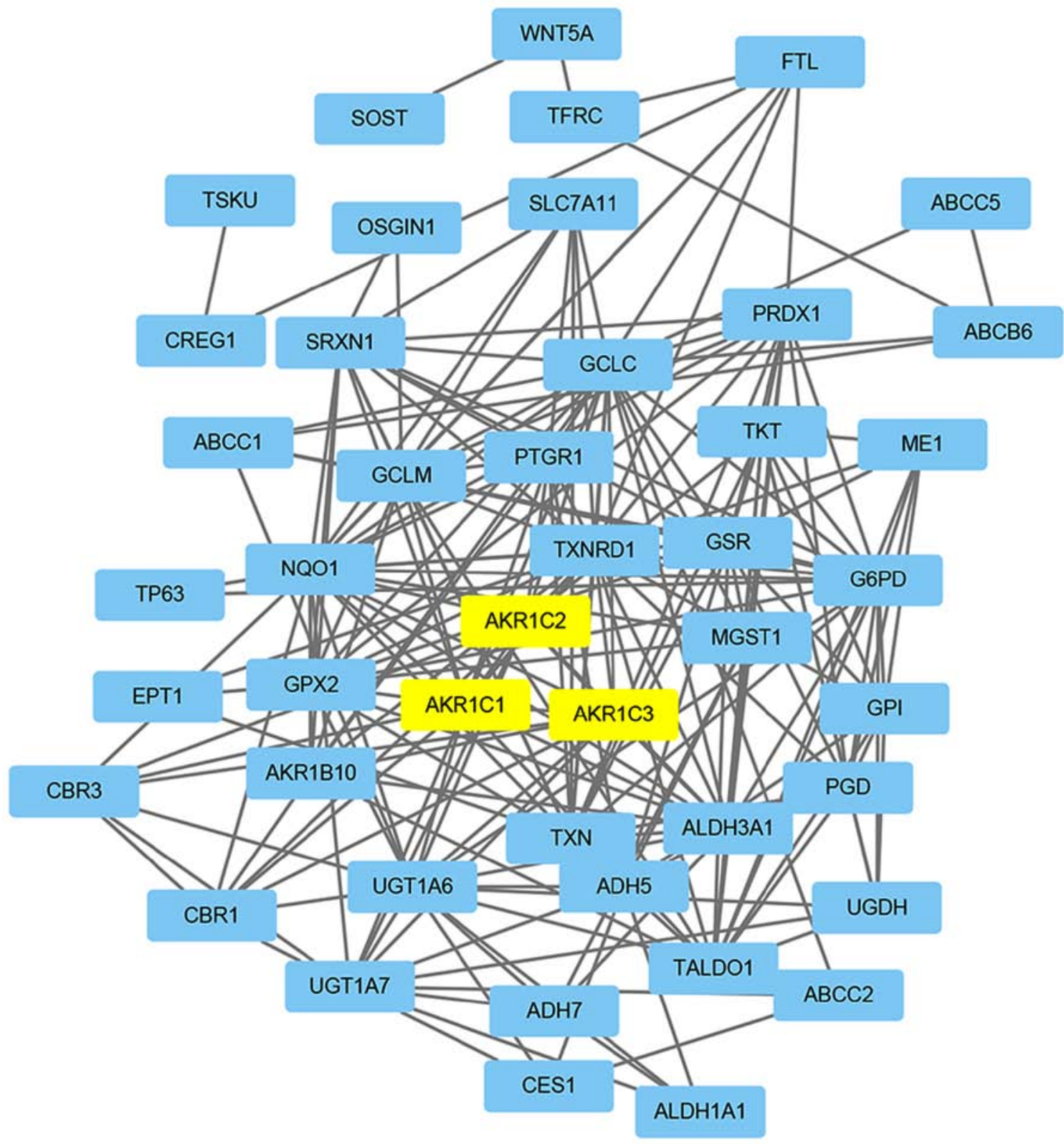

Figure 5. AKR1C1-3 are involved in a pathway with 44 unique interactors. Data were obtained from the Gene Expression Profiling Interactive Analysis database (http://gepia.cancer-pku.cn/), analyzed using the Search Tool for the Retrieval of Interacting Genes database (http://string-db.org/) and visualized using Cytoscape software. AKRIC1-3, aldo-keto reductase type 1C1-3.

of $A K R 1 C 2$ and $A K R 1 C 3$ are associated with liver cancer progression $(28,33)$. Although the AKR1C1-3 enzymes are involved in the same pathway, the exact mechanism of their contribution to the occurrence, development and prognosis of liver cancer is still unknown.

AKR1C1-3 can act as promising biomarkers for liver cancer diagnosis and prognosis. The high expression of AKR1C1-3, notably AKRIC3 may be associated with the incidence, development and prognosis of liver cancer. Nevertheless, the current study presented results derived solely from bioinformatics analyses and further experimental evidence is required to validate these findings.

\section{Acknowledgements}

Not applicable.

\section{Funding}

No funding was received.

\section{Availability of data and materials}

The datasets used and/or analyzed during the present study are available from the corresponding author on reasonable request.

\section{Authors' contributions}

SZ conceived and designed the study. SW, ZZ and WL made substantial contributions to the design of the current study, acquisition of data, interpretation of data and revising the manuscript. All authors read and approved the final manuscript. 


\section{Ethics statement and consent to participate}

Not applicable.

\section{Patient consent for publication}

Not applicable.

\section{Competing interests}

The authors declare that they have no competing interests.

\section{References}

1. Siegel RL, Miller KD and Jemal A: Cancer statistics, 2018. CA Cancer J Clin 68: 7-30, 2018

2. Heimbach JK, Kulik LM, Finn RS, Sirlin CB, Abecassis MM, Roberts LR, Zhu AX, Murad MH and Marrero JA: Aasld guidelines for the treatment of hepatocellular carcinoma. Hepatology 67: 358-380, 2018.

3. Liu S, Miao R, Zhai M, Pang Q, Deng Y, Liu S, Qu K, Liu C and Zhang J: Effects and related mechanisms of serotonin on malignant biological behavior of hepatocellular carcinoma via regulation of Yap. Oncotarget 8: 47412-47424, 2017.

4. Black AP and Mehta AS: The search for biomarkers of hepatocellular carcinoma and the impact on patient outcome. Curr Opin Pharmacol 41: 74-78, 2018.

5. Behne T and Copur MS: Biomarkers for hepatocellular carcinoma. Int J Hepatol 2012: 859076, 2012.

6. Zhao YJ, Ju Q and Li GC: Tumor markers for hepatocellular carcinoma. Mol Clin Oncol 1: 593-598, 2013

7. Barski OA, Mindnich R and Penning TM: Alternative splicing in the Aldo-Keto reductase superfamily: Implications for protein nomenclature. Chem Biol Interact 202: 153-158, 2013.

8. Rizner TL and Penning TM: Role of Aldo-Keto reductase family 1 (AKR1) enzymes in human steroid metabolism. Steroids 79 49-63, 2014

9. Frycz BA, Murawa D, Borejsza-Wysocki M, Wichtowski M Spychała A, Marciniak R, Murawa $P$, Drews $M$ and Jagodziński PP: Transcript level of AKR1C3 is down-regulated in gastric cancer. Biochem Cell Biol 94: 138-146, 2016.

10. Sun SQ, Gu X, Gao XS, Li Y, Yu H, Xiong W, Yu H, Wang W, $\mathrm{Li} \mathrm{Y}$, Teng Y and Zhou D: Overexpression of AKR1C3 significantly enhances human prostate cancer cells resistance to radiation. Oncotarget 7: 48050-48058, 2016.

11. Lewis MJ, Wiebe JP and Heathcote JG: Expression of progesterone metabolizing enzyme genes (AKR1C1, AKR1C2, AKR1C3, SRD5A1, SRD5A2) is altered in human breast carcinoma. BMC Cancer 4: 27, 2004.

12. Wenners A, Hartmann F, Jochens A, Roemer AM, Alkatout I, Klapper W, van Mackelenbergh M, Mundhenke C, Jonat W and Bauer M: Stromal markers AKR1C1 and AKR1C2 are prognostic factors in primary human breast cancer. Int J Clin Oncol 21: 548-556, 2016.

13. Rhodes DR, Yu J, Shanker K, Deshpande N, Varambally R, Ghosh D, Barrette T, Pandey A and Chinnaiyan AM: ONCOMINE: A cancer microarray database and integrated Data-Mining platform. Neoplasia 6: 1-6, 2004.

14. Barretina J, Caponigro G, Stransky N, Venkatesan K, Margolin AA, Kim S, Wilson CJ, Lehár J, Kryukov GV, Sonkin D, et al: The cancer cell line encyclopedia enables predictive modelling of anticancer drug sensitivity. Nature 483: 603-607, 2012

15. Győrffy B, Surowiak P, Budczies J and Lánczky A: Online survival analysis software to assess the prognostic value of biomarkers using transcriptomic data in Non-small-cell lung cancer. PLoS One 8: e82241, 2013.

16. Győrffy B, Lánczky A and Szállási Z: Implementing an online tool for genome-wide validation of survival-associated biomarkers in ovarian-cancer using microarray data from 1287 patients. Endocr Relat Cancer 19: 197-208, 2012.
17. Menyhart O, Nagy A and Gyorffy B: Determining consistent prognostic biomarkers of overall survival and vascular invasion in hepatocellular carcinoma. R Soc Open Sci 5: 181006, 2018.

18. Szász AM, Lánczky A, Nagy Á, Förster S, Hark K, Green JE, Boussioutas A, Busuttil R, Szabó A and Győrffy B: Cross-validation of survival associated biomarkers in gastric cancer using transcriptomic data of 1,065 patients. Oncotarget 7: 49322-49333, 2016.

19. Györffy B, Lanczky A, Eklund AC, Denkert C, Budczies J, Li Q and Szallasi Z: An online survival analysis tool to rapidly assess the effect of 22,277 genes on breast cancer prognosis using microarray data of 1,809 patients. Breast Cancer Res Treat 123: 725-731, 2010

20. Szklarczyk D, Franceschini A, Wyder S, Forslund K, Heller D, Huerta-Cepas J, Simonovic M, Roth A, Santos A, Tsafou KP, et al: STRING v10: Protein-protein interaction networks, integrated over the tree of life. Nucleic Acids Res 43 (Database Issue): D447-D452, 2015.

21. Chen X, Cheung ST, So S, Fan ST, Barry C, Higgins J, Lai KM, Ji J, Dudoit S, Ng IO, et al: Gene expression patterns in human liver cancers. Mol Biol Cell 13: 1929-1939, 2002.

22. Wurmbach E, Chen YB, Khitrov G, Zhang W, Roayaie S, Schwartz M, Fiel I, Thung S, Mazzaferro V, Bruix J, et al: Genome-wide molecular profiles of HCV-induced dysplasia and hepatocellular carcinoma. Hepatology 45: 938-947, 2007.

23. Roessler S, Jia HL, Budhu A, Forgues M, Ye QH, Lee JS, Thorgeirsson SS, Sun Z, Tang ZY, Qin LX and Wang XW: A unique metastasis gene signature enables prediction of tumor relapse in early-stage hepatocellular carcinoma patients. Cancer Res 70: 10202-10212, 2010 .

24. Tang Z, Li C, Kang B, Gao G, Li C and Zhang Z: GEPIA: A web server for cancer and normal gene expression profiling and interactive analyses. Nucleic Acids Res 45: W98-W102, 2017.

25. Hevir N, Vouk K, Sinkovec J, Ribic-Pucelj M and Rizner TL: Aldo-Keto reductases AKR1C1, AKR1C2 and AKR1C3 may enhance progesterone metabolism in ovarian endometriosis. Chem Biol Interact 191: 217-226, 2011.

26. Tian H, Li X, Jiang W, Lv C, Sun W, Huang C and Chen R: High expression of AKR1C1 is associated with proliferation and migration of small-cell lung cancer cells. Lung Cancer (Auckl) 7: 53-61, 2016.

27. Tiryakioglu NO and Tunali NE: Association of AKR1C3 Polymorphisms with bladder cancer. Urol J 13: 2615-2621, 2016.

28. Lu D, Zhang X and Cao X: The effect of AKR1C2 gene on hepatocarcinogenesis and its abnormal expression in hepatocellular carcinoma from Qidong, China, a liver cancer high risk area. Progress Biochemistry Biophysics 30: 906-918, 2003.

29. Zhong T, Xu F, Xu J, Liu L and Chen Y: Aldo-Keto reductase $1 C 3$ (AKR1C3) is associated with the doxorubicin resistance in human breast cancer via PTEN loss. Biomed Pharmacother 69: $317-325,2015$.

30. Xiong W, Zhao J, Yu H, Li X, Sun S, Li Y, Xia Q, Zhang C, He Q, Gao X, et al: Elevated expression of AKR1C3 increases resistance of cancer cells to ionizing radiation via modulation of oxidative stress. PLoS One 9: e111911, 2014.

31. Zeng CM, Chang LL, Ying MD, Cao J, He QJ, Zhu H and Yang B: Aldo-Keto Reductase AKR1C1-AKR1C4: Functions, regulation, and intervention for Anti-cancer therapy. Front Pharmacol 8: 119, 2017.

32. Dhanasekaran R, Limaye A and Cabrera R: Hepatocellular carcinoma: Current trends in worldwide epidemiology, risk factors, diagnosis, and therapeutics. Hepat Med 4: 19-37, 2012.

33. Abbattista MR, Jamieson SM, Gu Y, Nickel JE, Pullen SM, Patterson AV, Wilson WR and Guise CP: Pre-clinical activity of PR-104 as monotherapy and in combination with sorafenib in hepatocellular carcinoma. Cancer Biol Ther 16: 610-622, 2015.

This work is licensed under a Creative Commons Attribution-NonCommercial-NoDerivatives 4.0 International (CC BY-NC-ND 4.0) License. 